\title{
Fundamental Ideas and Objectives of Mathematical Education
}

\author{
Lead paper by Professor G. Polya, Ph.D. (Maths), \\ Stanford University, California, U.S.A.
}

\section{Introduction}

1. I am deeply indebted to the organizers of this Commonwealth Conference on Mathematics in Schools for having invited me to present the lead paper in this first session. I regard this invitation as a great honour, a great challenge, and a great opportunity. Yet, I must confess, I am also very much embarrassed by it. I see great difficulties inherent in my task and I don't know whether I am good enough to master them.

2. First of all, the aim of this conference is eminently practical. Each of us has urgent practical problems at home, and expects some contribution from this conference to their solution. Yet the theme of today's session is: "Fundamental ideas and objectives of mathematical education". You may have the impression that this theme is too remote from the concrete practical difficulties you have at home.

3. Secondly, I am embarrassed because you may expect me to tell you about the latest results of the science of education. Now, I must confess that I do not believe that there is such a thing as "the science of education". In my opinion teaching is not a science but an art. Or, let me put it a little more carefully (there is no time for a very careful statement): Teaching is, for the time being, much more an art than a science. Yet, if this is so, I cannot tell you scientific truths, just my personal opinions and it is embarrassing for a mathematician to assert things he cannot prove.

4. Here I am, however, and, as far as I can see, I can do nothing better than tell you my personal opinions which I acquired in doing mathematics, in teaching mathematics, and in thinking about the ways of doing and teaching mathematics for a good bit more than half a century. I would be glad, of course, if I could find among you kindred souls who hold similar opinions. Yet it is, in fact, more important to rouse those among you who have different opinions, because the task of this opening address is to start a debate.

5. I must discuss generalities, but I shall try to avoid empty generalities and keep as close to more concrete practical questions as I can. Generalities are needed to put the details into the right perspective. I wish to present you a "philosophy", but I shall emphasize simple points and obvious common sense. After all, I am not quite a philosopher. Do you know what a philosopher is? A philosopher knows EVERYTHING but nothing else. 


\section{General objectives of the School}

6. The family sends the child to the state supported school. What should the school do? The state, the family, the neighbours, the public opinion, everybody agrees, sometimes even the child himself: The school should enable the child to have a job, to earn a living. So it is in the United States, in other highly industrialized capitalistic countries, even in communistic countries.

7. In any social system the child should develop into an adult who can take care of himself and is well adapted to the community. The task of the school is to contribute its share to the child's development. In a very primitive and very stable community there is no need of schools: The child is sufficiently educated at home and by unplanned contacts with other members of the tribe. In general, the more complex the community's economic structure and the more rapid its technological or social change, the greater becomes the share of the school in the child's education. In the United States there is a definite trend: The young people stay in school longer and longer and the taxpayers vote higher and higher sums for the schools. And we can observe a similar trend almost everywhere.

8. To turn the child into an employable adult is a crude and narrow aim. In fact, if we conceive this aim too narrowly we are almost bound to miss it. Observe that in a complex economic system there are many different kinds of jobs. The individual should find the job for which he is the most suitable; this is not only in his interest but also in the interest of the community. Yet, to choose the most suitable he must know all the possibilities and so he must have some knowledge of the whole world around him, some sort of general culture.

9. Moreover, to fill his job well, he must be well developed. Yet we do not know in advance what kind of job the growing child will eventually have, and so we must develop him as far as possible in all respects.

10. And so, beyond the crude and narrow objective "turn out employable adults", there appear higher and wider objectives of the school: Develop all the inner resources of the child. Give him general culture.

11. I think that the old Greek philosopher Plato would not disapprove of these wider aims. I hope that you don't disapprove of them.

\section{Narrow objectives of mathematical education}

12. In an economic system that is above a certain primitive level analphabets are scarcely employable. Hence there arises the obvious need of a primary school that should teach every child the three R's, reading, writing, arithmetic. Let me use (in this general introductory talk) the term "primary school" in a not too sharply circumscribed sense which is roughly the following: The school that every child of the age 6 to 12 should attend. To teach the rudiments of arithmetic (the natural numbers and the four basic operations with natural numbers) is an obvious crude minimum task of the primary school. Let me add two items which are almost as necessary and then we arrive at a somewhat enlarged, but still very modest aim of primary mathematical education: 
Primary narrow objective:

(1) Arithmetic of natural numbers $(+,-, \times, \div)$

(2) Length, area, volume

(3) Fractions, percentage

Also the term "secondary school" should be used in this talk in a not too sharply defined sense: A school that some children of the age 12 to 18 should attend. And let us consider a corresponding modest aim of mathematical education:

Secondary narrow objective:

Professional preparation of prospective technical personnel (technicians, engineers, scientists, managers).

13. Let me emphasize that the two objectives just formulated are severely restricted, narrow, minimum goals, dictated by obvious economic needs. I think that there is little doubt that the schools should attain these narrow objectives. Yet I think too, and I hope that most of you will agree, that the schools should do more and also attain some wider and higher objectives.

14. Let me point out that those narrow objectives themselves may lead to certain wider objectives.

15. There is no doubt that the primary school should teach arithmetic. Now, for all practical purposes, it is sufficient that people do their arithmetic mechanically; only some speed and accuracy matters. Yet the school should do more: We should teach the children to do their arithmetic insightfully. Why? Because teaching them so we may get results faster and more permanent results. Insightful knowledge is a more ambitious aim than mechanical knowledge. Yet, in teaching as in other activities, the more ambitious aim may have more chances of success.

16. Now, let us look at the secondary school. Mathematics above the primary level is strictly mandatory, immediately necessary only for prospective users of mathematics, for certain kinds of technical personnel. Yet when the child enters the secondary school we don't know yet whether he will or will not exercise a technical profession later and so we are obliged to teach mathematics to all entrants. Now, it would be a sad thing if the future non-technicians, who may be the majority, would derive no benefit from years of mathematical study. Therefore we must find a wider objective for the teaching of mathematics in the secondary school so that it should of fer something to both kinds of students, to those who will, and to those who will not, use mathematics in their later studies or profession.

\section{General Wider Objectives}

17. I have tried hard to show you that starting from narrowly restricted goals of education we may arrive at wider objectives and higher ideals:

Serve the individual and the community.

Develop all the inner resources of the child.

Several such general objectives of education have been proposed, from Plato downward, in various formulations. Here are a few: 
General culture.

Discipline of the mind.

Desirable habits of thinking.

Mental and emotional maturity.

A well balanced personality.

Which one of these aims do you prefer? Which one deserves to be preferred?

18. I don't know. I think that all these aims are respectable, I believe that they are all desirable, but I don't know which one is the most desirable - and if I did know I would not take the time to discuss it here. Because, up to a certain point, it does not matter. These objectives certainly overlap a good deal and even those sounding rather different may lead, if reasonably interpreted, to the same practical consequences.

19. Each aim mentioned embodies some high ideal worth striving for. Yet every one may be worthless if you pay only lip service to it, and it may be worse than worthless if it is used as a cheap and empty slogan by pretentious incompetents who are usually the more pretentious the more incompetent they are. Each of those ideals is worthwhile provided that you honestly believe in it. And it is really valuable if you earnestly try to live up to it, if you translate it into the everyday practice of the school.

20. Your ideal, whatever it is, should be somehow present in your mind whenever you are planning the curriculum, or preparing yourself for the next lesson, or choosing a problem for homework. Does the item you are considering contribute to some practical, clearly defined narrow objective? Or does it contribute to some wider objective such as general culture or mental discipline? If it does not, why should you bother or why should you bother the children with it? A child in your class or his father could ask you: "But, teacher, what is it good for?" Can you answer this question to your own satisfaction?

21. My opinion is: Have some ideal, see the details of your profession in the light of your ideal, and realize that any detail may raise the question "What is it good for?" Then you will become a better teacher.

\section{On learning: Two simple rules}

22. I have to say now a few words on the process of learning. This is a subject about which old philosophers and modern psychologists said and wrote and printed more than any one of us could read. Yet whatever we read we should read with attention and accept only what we can confirm on the basis of our own well digested experience.

23. I wish to concentrate upon two points which a teacher should never disregard and which may easily arise in our later discussion. They are simple, can be formulated in everyday language, they are essentially classical, and pretty generally, if not universally, accepted. I will call them "principles of learning" but if you wish to call them "rules of thumb" instead of "principles" I shall not be offended at all. 
24. (1) Active learning. It has been said again and again that learning should be active, not merely passive or receptive. It has also been said, along the same line, that the best way to learn anything is to discover it by yourself. This idea is very old, it can be traced back to Socrates who expressed it by a picturesque metaphor: The ideas should be born in the student's mind and the teacher should act only as midwife.

Yet time is limited, the teacher cannot wait when the labor of childbirth is too long and difficult. Moreover, we cannot expect that high school kids will rediscover the whole of human science, and so we must settle for something less: Let the students discover by themselves as much as feasible under the given circumstances.

(2) Consecutive phases. It has been said again and again that, in learning, things should come before words, the concrete before the abstract, doing and seeing before verbal expression, and so on. I think that you can find quotations to some such effect in the writings of all famous educators, from Comenius to Montessori and after, but let me quote just one, a sentence by Kant: Thus all human cognition begins with intuitions, proceeds from thence to conceptions, and ends with ideas.

It seems to me that this sentence suggests at least some, if not all, of the more important priorities to which we, as teachers, must pay attention. Yet let me paraphrase it, restate it in more down-to-earth language: Learning begins with action and perception, proceeds from thence to words and concepts, and should end in desirable mental habits.

I hope that this sentence touches the right chord with you. To explain it thoroughly would need close consideration of several examples for which we obviously have no time. I believe, however, that the two rules formulated here can yield valuable guidelines to teachers who understand them seriously, intimately, on the basis of their own well considered personal experience.

\section{On problems, problem-solving, and the tactics of problem-solving}

25. I expect that you have essentially known all or most of the objectives of education and mathematical education and also the principles of learning $I$ have mentioned so far, but I thought that it might be useful to remind you of them and emphasize such points as may arise in one way or another in our later discussions.

26. I am glad that I can say something less well-known and more personal about the next topic.

(a) From any one of the general objectives mentioned you can get down to concrete details of the curriculum in many different ways: There is no scientifically guaranteed best way of teaching, there are as many good ways of teaching as there are good teachers.

Yet there is one specific point about which there is little dissension: All experts agree that mere possession of information is of little value in mathematics. To know mathematics means to be able to do mathematics: 
To use mathematical language, to find the unknown, to check a proof, and so on. Therefore, to teach mathematics we must give opportunity to the student to do mathematics.

There are many ways of doing mathematics but, from several viewpoints, solving problems appears as the most cardinal mathematical activity. "The solution of problems is the most characteristic and peculiar sort of voluntary thinking", wrote the renowned American psychologist William James. In fact, solving problems may be considered as the specific achievement of intelligence, and intelligence is the specific gift of mankind. Adults are working at their problems and worrying about them, and children's play, which is an anticipation of adult life, is often a sort of problem-solving. Solving mathematical problems at their level comes early and quite spontaneously to some children, and I think that all children could be made ready for it pretty early by the right approach.

At any rate, higher mathematical activities (such as framing new concepts, building theories, constructing axiomatic systems) presuppose considerable mathematical experience which must be acquired mainly by solving problems.

Hence it is well justified that all mathematical textbooks, beginning with the Rhind papyrus, contain problems. The problems may even be regarded as the most essential contents of a textbook and problemsolving by the students as the most essential part of mathematical instruction.

(b) Yet "problem-solving", which became a sort of slogan recently, means different things to different people.

One thing is to teach a specific procedure to solve a specific kind of problem, for instance the usual formula to solve quadratic equations. This may be useful for certain students who need such specific skill in their future profession or in later studies, perhaps for an examination yet it is hardly useful in itself for the general student. Why should the future lawyer or the future truckdriver solve quadratic equations?

A very different thing is to let the students do mathematical problems in the hope that desirable mental habits will result from their work, such as orderliness, precision, ability to concentrate, ability to handle abstract concepts, to name some of the more popularly known objectives of this kind.

There are problems and problems. We must be especially concerned here with the difference between routine problems and non-routine problems.

A routine problem has a specific aim, it should teach the student to use correctly this or that particular rule or procedure or definition, it offers drill and practice, and it does not demand any invention or originality. To solve a quadratic equation with given numerical coefficients is a routine problem for a student who has been shown the general formula.

On the contrary, a non-routine problem challenges the student's inventiveness and originality, it should aim at some more general and 
higher objective, and I believe that no higher objective of secondary mathematical education can be attained without the judicious use of non-routine problems.

I must confess to you that I feel uncomfortable when I have to listen to a speaker on problem-solving who does not discriminate between routine and non-routine problems. And I feel particularly uncomfortable when the whole behaviour of the speaker arouses the suspicion that he has never solved a non-routine problem himself.

Yet I may be prejudiced: Non-routine problems are crucial for that objective of secondary mathematical education that I personally prefer to all others. My favourite aim is: Mathematical problems should be used to implant in the students' minds whatever attitudes and procedures may be generally useful for solving any kind of problem, the tactics of problem-solving.

(c) I have now arrived at my favourite topic. Of course, I would like to talk about it a lot, but there is time only for a brief and rather imperfect sketch.

In teaching problem-solving in the mathematics class we quite naturally come across attitudes and patterns of thought, the usefulness of which is not restricted to mathematical problems.

You all know that widespread bad habit of students: When they have to solve a problem, especially in an examination, they dive into computations or constructions without having quite realized what the proposed problem actually is. Yet the right attitude is just the opposite: First of all, understand your problem. Distinguish its principal parts and see each of them as clearly as you can. Try to foresee the result - there is perhaps an easy way to estimate the unknown. Yet, above all, try to conceive a plan before you dive into details.

What I have tried to describe here is an attitude the usefulness of which extends far beyond elementary mathematical problems, yet we can impress it on the students' minds in the mathematics class. This attitude (which would deserve a more careful and more detailed description illustrated by example) belongs to the tactics of problem-solving.

I have trouble tearing myself away from this topic. Yet let me just mention a problem-solving procedure, developed on elementary mathematical problems, the interest of which, however, extends far beyond them. Its discovery was attributed to the philosopher Plato by some ancient authors. It was certainly developed by the Greek geometers who called it "analysis" which means "solution backward" in Greek. As the word "analysis" is used today in several different meanings it is preferable to use some other term such as "regressive argument" or simply "working backward". The procedure is mentioned in most older textbooks of geometry: We begin by "taking for granted" the result, the conclusion that we have to prove or the figure that we have to construct. Then we derive from it some other conclusion or figure, hence still 
another one, and so on, until we arrive at the hypothesis or the data proposed. A fuller description illustrated by appropriate examples would show that this procedure of "working backward" is by no means restricted to geometry, but has a wider interest; it belongs in fact to the tactics of problem-solving.

The foregoing examples, to which I am not allowed to add more here, should give you a first idea of the "tactics of problem-solving". 1

It is my conviction which I wish to express here as strongly as I can: The teaching of mathematics in the secondary school should emphasize the tactics of problem-solving.

Some of the students will, others will not, use mathematics after leaving the secondary school. For users of mathematics the tactics of problemsolving may be the beginning of their professional attitude. For non-users it may easily be the most useful thing that remains with them from the mathematics of the secondary school.

There is much more to say about problems, problem-solving, the tactics of problem-solving, and the relation of these things to the general objectives of mathematical education and the curriculum. Perhaps there will be an opportunity later, in a working group, for a conversation on the details of certain questions raised.

\section{Conclusion}

27. The time has arrived for looking back at the discussion of the past hour.

I have talked to you about problems, problem-solving, and the tactics of problemsolving. Yet I did not urge you to adopt my views. If there was anything in what I have said that will start you reconsidering this or that point of your teaching it will be enough and a great satisfaction to me.

28. My views on problem-solving in the schools spring from a general outlook which you may call my "educational philosophy". It is a very informal, down-toearth philosophy and I presented it, or, rather, hinted it, to you very informally just "between the lines". And certainly I did not urge you to adopt it. In teaching, you see, as in many other things, it does not matter much what your philosophy is; it matters more that you have a philosophy; but it matters very much whether you do or don't live up to your philosophy.

29. May I repeat it: I hope that there is some higher objective of your teaching and that you try to see the details of your everyday work in the light of that higher objective. When some item comes up for decision, your first question must be, of course: Does it serve some narrow objective prescribed by the needs of the community? If it does not, what is it good for? Does it serve your wider and higher objective?

30. It would help, I think, if we approached the coming discussions of this conference in the spirit of these questions.

1 Which is better called "heuristics" in other contexts: I used the latter term in my books:

How to Solve It, Princetown University Press, 1945; 2nd edition, Doubleday and Co., 1957.

Mathematics and Plausible Reasoning, vol. 1 and 2, Princetown University Press, 1954.

Mathematical Discovery, vol. 1 and 2, John Wiley and Sons, 1962/65. 